\section{IUPAC Glossary of Terms Used in Immunotoxicology (IUPAC Recommendations 2012)}

D.M. Templeton, M. Schwenk, R. Klein, and J.H. Duffus

Pure and Applied Chemistry, 2012

Vol. 84, No. 5, pp. 1113-1295

The primary objective of this "Glossary of Terms Used in Immunotoxicology" is to give clear definitions for those who contribute to studies relevant to immunotoxicology but are not themselves immunologists. This applies especially to chemists who need to understand the literature of immunology without recourse to a multiplicity of other glossaries or dictionaries. The glossary includes terms related to basic and clinical immunology, insofar as they are necessary for a selfcontained document, and terms related to diagnosing, measuring, and understanding effects of substances on the immune system. The glossary consists of about 1200 terms as primary alphabetical entries, annexes of common abbreviations, examples of chemicals with known effects on the immune system, autoantibodies in autoimmune disease, and therapeutic agents used in autoimmune disease and cancer. The authors hope that among the groups that will find this glossary helpful, in addition to chemists, are toxicologists, pharmacologists, medical practitioners, risk assessors, and regulatory authorities. In particular, it should facilitate the worldwide use of chemistry in relation to occupational and environmental risk assessment.

http://dx.doi.org/10.1351/PAC-REC-11-06-03

\section{Names and Symbols of the Elements with Atomic Numbers 114 and 116 (IUPAC Recommendations 2012)}

Robert D. Loss and John Corish

Pure and Applied Chemistry, 2012

Vol. 84, No. 7, pp. 1669-1672

A joint IUPAC/IUPAP Working Party (JWP) has confirmed the discovery of the elements with atomic numbers 114 and 116. In accordance with IUPAC procedures, the discoverers proposed names as follows: flerovium with the symbol $\mathrm{Fl}$ for the element with $Z=114$ and livermorium with the symbol Lv for the element with $Z=116$. The IUPAC Inorganic Chemistry Division recommended these proposals for acceptance, and they were adopted on 23 May 2012 by the IUPAC Bureau as delegated to act by the IUPAC Council meeting on 3-4 August 2011.

http://dx.doi.org/10.1351/PAC-REC-11-12-03

Analogue-Based Drug Discovery: Contributions to Medicinal Chemistry Principles and Drug Design Strategies. Microtubule Stabilizers as a Case in Point (special topic article)

Mohammad H. El-Dakdouki and Paul W. Erhardt Pure and Applied Chemistry, 2012

Vol. 84, No. 7, pp. 1479-1542

The benefits of utilizing marketed drugs as starting points to discover new therapeutic agents have been well documented within the IUPAC series of books that bear the title Analogue-based Drug Discovery (ABDD). Not as clearly demonstrated, however, is that ABDD also contributes to the elaboration of new basic principles and alternative drug design strategies that are useful to the field of medicinal chemistry in general. After reviewing the ABDD programs that have evolved around the area of microtubule-stabilizing chemo-therapeutic agents, the present article delineates the associated research activities that additionally contributed to general strategies that can be useful for prodrug design, identifying pharmacophores, circumventing multidrug resistance, and achieving targeted drug distribution.

\section{http://dx.doi.org/10.1351/PAC-CON-12-02-13}

